\title{
The Application of GARCH Model in The Growth Enterprise Market Risk Measurement
}

\author{
Qian Rao \\ College of Economics and Management \\ Hebei University of Science and Technology \\ Shijiazhuang, Hebei, China \\ e-mail: raoqian100@163.com
}

\author{
Zan Zhang \\ College of Economics and Management \\ Hebei University of Science and Technology \\ Shijiazhuang, Hebei, China \\ e-mail: zhangzan0512@163.com
}

\begin{abstract}
After the financial crisis, many countries have been paying more and more attention to the regulation of financial market risk, and the core of risk regulation is the market risk measurement. Based on the theory of risk management, this paper studies the statistical characteristics of the Growth Enterprise Market (GEM) index, do the empirical researches of the GEM composite index with the VaR and GARCH model, and the results show that the VaR value risk measurement method based on the GARCH model group can accurately measure the market risks of GEM in China.
\end{abstract}

Keywords-Growth Enterprise Market; GARCH model; VaR; Risk measurement

\section{INTRODUCTION}

Entering the 21st century, the paces of financial innovation and information technology progress are accelerating, and the rapid development of the global financial industry brings strong driving force for the development of world economy. The Chinese financial market scale has been increasing rapidly since the establishment of the Shanghai and Shenzhen Stock Exchange in the 1990s. In order to enrich the market trade category, and provide listed financing channels for small and medium-sized enterprises, China's GEM is launched on October 30, 2009. The GEM is an important part of the Chinese securities market, which raise a lot of money for the development of small and medium-sized enterprises and strategic emerging industries, and significantly increase the social invested capital.

However, the volatility of global financial market has also intensified especially in the period of global financial tsunami which causes great loss to the world economy. The financial crisis also makes people recognize the importance of financial market's risk control. The key of risk management is risk measurement, which directly lead to the success or failure of risk regulation. Therefore, using a reasonable method to measure the market risk of GEM in China is one of the effective measures to prevent financial risks.

At present, the method of Value at Risk (VaR) based on the Generalized Autoregressive Conditional Heteroskedasticity $(\mathrm{GARCH})$ model has become the most popular method to measure the market risk for the GARCH model can better describe the dynamic characteristics of returns fluctuations in stock market, and capture the clustering and asymmetric features of stock market. In the GARCH model, we need make an assumption that the returns follow a probability distribution. For the normal distribution can't reflect the sharp peak and fat-tailed features of returns series, many scholars did some research about the $t$ distribution and Generalized Error Distribution (GED), and the results show that $t$ distribution and GED can better reflect the characteristics of the return series.

Based on the studies at home and abroad, in this paper, we analyze the market risk in GEM by building GARCH and VaR model under the different distribution assumption with the latest data of GEM composite index in China. As a result, we calculate and compare the $\mathrm{VaR}$ values got from different distributions and models, and discover the model which is most suitable for the market risk management of GEM in China.

\section{VAR METHOD AND GARCH MODEL}

\section{A. Value at Risk}

The VaR value is the biggest loss that an asset or group of assets may occur within a particular time in the future under the conditions of normal fluctuations in market and the given confidence level, and was first proposed by J.P.Morgan company. Under the Assumptions that the confidence level is $\alpha$, the loss of asset or portfolio of assets in holding period is $\Delta p$, so the definition of the VaR can be expressed as:

$$
\operatorname{prob}(\Delta p>V a R)=1-\alpha
$$

In general, with the concrete distribution, defined $W_{0}$ as the initial investment, $\mathrm{R}$ is the expected return ,the volatility of $\mathrm{R}$ is $\sigma$, and $R^{*}$ represents the minimum return rate under a certain confidence level, the value of portfolio and minimum investment value at the end of holding period are, $W=W_{0}(1+R)$ and $W^{*}=W_{0}\left(1+R^{*}\right)$ respectively, and the $\mathrm{VaR}$ of a portfolio can be shown as:

$$
\operatorname{VaR}=E(W)-W^{*}=W_{0}\left(R-R^{*}\right)
$$

From the above formula, we can calculate the $\mathrm{VaR}$, we get the smallest investment value of the portfolio or the minimum return rate of investment,. We can get $W^{*}$ and $R^{*}$ from the formula below: 


$$
\alpha=\int_{-\infty}^{w^{*}} f(w) d w=\int_{-\infty}^{R^{*}} f(r) d r
$$

Where $f(w)$ and $f(r)$ are the probability distribution of portfolio's value and invest returns.

Depending on whether the rate of return follows a certain distribution, the calculation method of $\mathrm{VaR}$ can be divided into three categories: parameter method (also called variance - covariance method), nonparametric methods (including historical simulation method and Monte Carlo simulation method), and semi-parametric methods (including extreme value theory, etc.). This paper uses the parameter method to calculate VaR, which has the assumption that the return series follow a certain distribution, such as normal distribution. Under the hypothesis of certain distribution, the VaR calculation formula can be shown as:

$$
V a R=W_{0} Z_{\alpha} \sigma \sqrt{\Delta t}
$$

Where $Z_{\alpha}$ is down quantile when the distribution probability is $\alpha, \Delta t$ is the holding period of assets.

\section{B. The test of VaR accuracy}

The accuracy test of VaR model refers to how many the measurement results of $\mathrm{VaR}$ model cover the actual loss. The accuracy of VaR model has multiple representations, therefore it has many test methods, and the common method is the failure frequency test proposed by Kupiec (1995). He assumed that the VaR estimation has time independence, the trial is a failure when the actual loss is more than VaR estimates, and the trial is a success when the actual loss is lower than the estimates of $\mathrm{VaR}$, so the binomial results of observation represent a series of independent Bernoulli trials, and the expected probability of failure is $p^{*}=1-c$ ( $c$ is confidence level). We assume that the confidence level of calculating $\mathrm{VaR}$ is $\alpha$, the number of actual inspection day is T, and the number of failed days is $\mathrm{N}$, and the failure frequency is $p(N / T)$.

The null hypothesis is $p=p^{*}$, so that the evaluation of VaR accuracy is transformed to whether the failure frequency $p$ is significantly different from $p^{*}$. Kupiec thought that the most appropriate test of null hypothesis is likelihood ratio test:

$$
L R=-2 \ln \left[\left(1-p^{*}\right)^{T-N} p^{* N}\right]+2 \ln \left[(1-p)^{T-N} p^{N}\right]
$$

Under the condition of null hypothesis, $L R$ statistics follow the $\chi^{2}$ distribution with one degree of freedom.

\section{The model of GARCH group}

\section{1) The GARCH model}

The process of GARCH model considers the feature of conditional heteroscedasticity owned by financial tools, so it can better depict the cluster effect of stock returns series. The GARCH (p, q) model includes two equations: one is the mean equation; the other is the conditional variance equation, which can be expressed in the following formula:

$$
\begin{gathered}
r_{t}=c \mu_{t}+\varepsilon_{t} \\
\sigma_{t}^{2}=\alpha_{0}+\alpha_{i} \sum_{i=1}^{q} \varepsilon_{t-q}^{2}+\beta \sum_{j=1}^{p} \sigma_{t-p}^{2}
\end{gathered}
$$

Where $\left\{r_{t}\right\}$ is the logarithm returns sequence, $c$ is the coefficient of mean equation, $\varepsilon_{t}$ is the random error term which is zero mean and independent identically distributed, $\sigma^{2}$ is conditional variance, $\varepsilon_{t}$ and $\sigma$ are independent of each other, $\alpha_{0}$ is the constant term, $\alpha_{i}$ is the coefficients of $\mathrm{ARCH}, \beta_{j}$ is the coefficients of GARCH.

\section{2) The GARCH-M model}

In financial applications, it is natural to assume that the expected return of assets is proportional to the expected risk, in another word, the greater the income, the greater the risk, so people would introduce the condition variance or standard deviation as exogenous variables into the mean equation. the GARCH model can be divided into general GARCH model and the GARCH - M (mean) model, according to the different conditions of mean equation, namely the mean regression equation. The model can be shown as follows:

$$
\begin{gathered}
r_{t}=c+\theta \sigma_{t}+\varepsilon_{t} \\
\sigma_{t}^{2}=\alpha_{0}+\alpha_{i} \sum_{i=1}^{q} \varepsilon_{t-q}^{2}+\beta \sum_{j=1}^{p} \sigma_{t-p}^{2}
\end{gathered}
$$

Here $\theta$ is the risk coefficient of mean equation. The main difference with general GARCH model is the mean equation consider the risk.

\section{3) The EGARCH model}

The movement of financial price has leverage effect, that the rise and fall of the stock price may affect the subsequent fluctuations asymmetrically. The fall of stock price have a greater impact on the subsequent movements than the same margin of rise, which means that the better model should make asymmetric reactions to plus or minus residual errors. In order to capture the asymmetry of positive and negative interference, and more accurately depict the stock volatility, Nelson put forward the Exponential GARCH (EGACH) model in 1991:

$$
\ln \sigma_{t}^{2}=\alpha_{0}+\sum_{j=1}^{\infty} \beta_{j} \ln \sigma_{t-1}^{2}+\sum_{i=1}^{q} \alpha_{i}\left|\frac{\varepsilon_{t-i}}{\sqrt{\sigma_{t-i}}}\right|+\theta_{i} \frac{\varepsilon_{t-i}}{\sqrt{\sigma_{t-i}}}
$$

The conditional variance of EGARCH model uses the natural logarithm, and presents the leverage effect of exponential. Another key feature of the model is the information is asymmetric; if $\theta<0$, the leverage effect is significant, and the negative impact causes a greater volatility than the positive impact in stock market.

\section{VAR ANALYSIS OF GROWTH ENTERPRISE MARKET}

\section{A. The statistical analysis of sample data}

In order to reflect the overall risk of the GEM, this paper selects the GEM composite index included all the stocks of GEM as the data sample. The base date of GEM composite index calculation is May 31, 2010, so we select the period sample data from June 1, 2010 to December 31, 2013, excluding the days on Saturday, Sunday and statutory holidays, and get 868 samples.

We adopt the logarithm returns form of GEM composite index as the research object, which is shown 
as $\gamma_{t}=\ln p_{t}-\ln p_{t-1}$, where $p_{t}$ is the day's closing price of index, and $p_{t-1}$ is the previous day's closing price of index.
In order to better describe the statistical characteristics of the GEM composite index (GCI), we select the same period of the Shanghai composite index (SCI), and the specific characteristics shown in TABLE I:

TABLE I. BASIC StATistical Characteristics of The GEM, MAin BoARd Stock IndeX

\begin{tabular}{|c|c|c|c|c|c|c|c|c|}
\hline Index & Mean & Maximum & Minimum & Std. Dev. & Skewness & Kurtosis & J-B value & Probability \\
\hline GCI & 0.000134 & 0.0273 & -0.038 & 0.00836 & -0.45 & 3.9026 & 58.81 & 0.000 \\
\hline SCI & 0.000098 & 0.01838 & -0.0236 & 0.00514 & -0.15 & 4.6153 & 97.68 & 0.000 \\
\hline
\end{tabular}

It is clear from the above table that the income of GEM composite index is greater than the Shanghai composite index, which reflects the high growth of listed companies in GEM; at the same time, the risk of GEM market is also higher than the main board market. The values of skewness and kurtosis in two markets deviated from the corresponding value of normal distribution ( 0 and 3 ), which means that the returns series have fat-tail feature. What's more, the J - B values of two indexes are far greater than the critical value of 5.992, which refuse to the assumption that the returns series follow the normal distribution, further confirm that the two markets has the characteristics of high peak and fat tail.

\section{B. The stationarity test}

We test the stationarity of returns series with unit root test, and the calculation results are shown in TABLE II. The $\mathrm{ADF}$ value is -21.6 , less than the critical values under $1 \%$,
$5 \%$ and $10 \%$ confidence level, so refuse to the null hypothesis that the sequence exist unit root, namely the the returns sequence of GEM index is significantly stable.

TABLE II.

ADF TEST RESULTS OF GEM INDEX

\begin{tabular}{|c|c|c|}
\hline & $\mathrm{t}-$ Statistic & Prob. $^{*}$ \\
\hline ADF Statistic & -21.64610 & 0.0000 \\
\hline $1 \%$ critical value & -3.437678 & \\
\hline $5 \%$ critical value & -2.864664 & \\
\hline $10 \%$ critical value & -2.568487 & \\
\hline
\end{tabular}

\section{The conditional heteroscedasticity test}

It can be found that the sequence has the feature of clustering obviously through GEM index returns line chart (Figure1), so we need to test the ARCH-LM heteroscedasticity effect of index returns series.

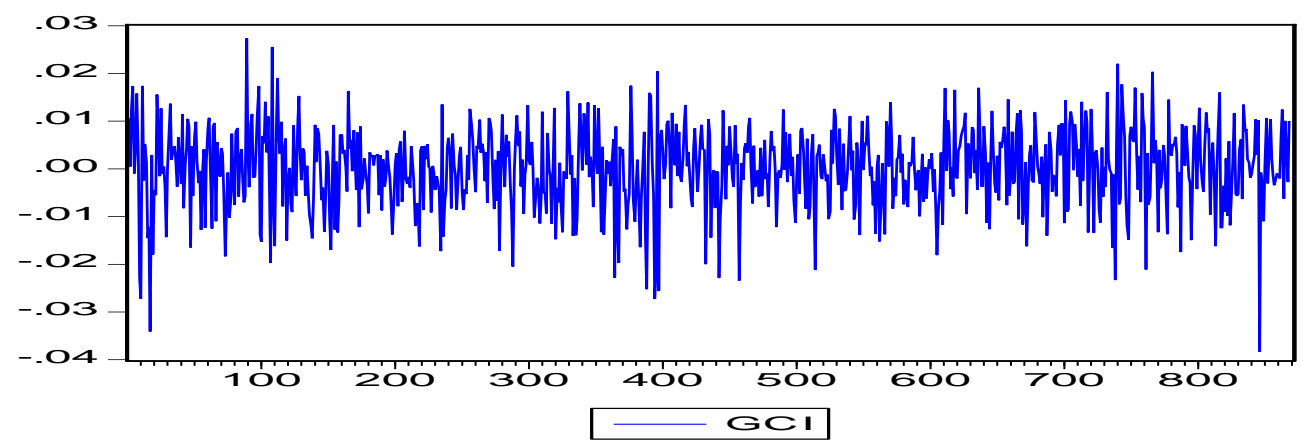

Figure 1. Line chart of GEM index returns

Then we calculate the Autocorrelation Coefficient (AC) and Partial Autocorrelation Coefficient (PAC) of GEM index returns sequence, and find that the sequence has significant autocorrelation with its first three order lag returns under 5\% significance level, and establish the regression model between them. At last, we use the ARCH-LM method test whether the residual of regression model exist the ARCH effect, and the ARCH - LM test results as shown in TABLE III.

TABLE III.

ARCH - LM TEST RESULTS

\begin{tabular}{|c|c|c|c|}
\hline F-statistics & 5.2643 & P value & 0.001 \\
\hline $\mathrm{N}^{*} R^{2}$ statistics & 15.580 & P value & 0.001 \\
\hline
\end{tabular}

statistic and $\mathrm{N} * R^{2}$ statistic in ARCH - LM test are 0.001 , which is less than the significance level of 0.05 , so we reject the null hypothesis that does not exist the $\mathrm{ARCH}$ effect, in other words, the residual error sequence exist ARCH effect.

\section{The caculation of VaR based on GARCH model}

The above analysis and test show that: the returns sequence of GEM composite index is stable, it doesn't follow the normal distribution, and has the features of fat tail and conditional heteroscedasticity significantly, therefore, we respectively build the GARCH, GARCH-M and EGARCH model of the residual error sequence to measure the volatility of returns sequence under the assumptions of normal distribution, $\mathrm{t}$ distribution and GED distribution. According to the AIC and SC information rules, we can build the $\operatorname{GARCH}(1,1)$ model group of residual sequence. TABLE IV shows the model parameters of GEM logarithm returns under different 
distributions.

TABLE IV

THE PARAMETER ESTIMATION RESUlts OF GARCH MOdEL GROUP

\begin{tabular}{|c|c|l|l|l|l|l|}
\hline \multirow{2}{*}{ Model } & Distribution & \multicolumn{1}{c|}{$\boldsymbol{C}$} & \multicolumn{1}{c|}{$\alpha_{0}$} & \multicolumn{1}{c|}{$\alpha_{1}$} & \multicolumn{1}{c|}{$\boldsymbol{\beta}$} & $\boldsymbol{\theta}$ \\
\hline \multirow{3}{*}{ GARCH } & normal & -0.031922 & $2.19 \mathrm{E}-06$ & 0.027695 & 0.939281 & \\
\cline { 2 - 7 } & $\mathrm{t}$ & 1.347765 & $2.64 \mathrm{E}-06$ & 0.03121 & 0.92904 & \\
\cline { 2 - 7 } & GED & 1.365215 & $2.46 \mathrm{E}-06$ & 0.028808 & 0.934236 & \\
\hline \multirow{3}{*}{ GARCH-M } & normal & -0.005703 & $2.55 \mathrm{E}-06$ & 0.035027 & 0.926905 & 0.705452 \\
\cline { 2 - 7 } & $\mathrm{t}$ & -0.006276 & $2.81 \mathrm{E}-06$ & 0.0358 & 0.922222 & 0.800768 \\
\cline { 2 - 7 } EGARCH & GED & -0.006209 & $2.72 \mathrm{E}-06$ & 0.035265 & 0.924176 & 0.792342 \\
\cline { 2 - 7 } & normal & 0.0000372 & -0.250605 & 0.059837 & 0.978888 & -0.002937 \\
\cline { 2 - 7 } & GED & 0.000216 & -0.29969 & 0.065616 & 0.974259 & -0.00364 \\
\hline
\end{tabular}

From the results of parameter estimation, we can see that all the three models can well reflect the clustering effect of returns series. The parameters of $\beta$ in the three models are between 0.9 and 1 , which means that the volatility has a strong memory, and the effect of early fluctuations on the current fluctuation presents attenuation trend. As to the EGARCH model, the leverage effect parameter- $\theta$ is negative, indicating that the volatility of returns series is obviously asymmetrical, and the fluctuation caused by negative impact is bigger than positive impact. The shape parameters of GED distribution based the three models are 1.68, 1.65 and 1.69 , respectively, all of them is less than 2 , which means that the GED distribution is suitable for describing the returns features of sharp peak and fat tail.

Through the parameter estimation of GARCH model, we forecast the standard deviation of returns sequence with the parameters got from the models. Then the VaR values of each day can be calculated under the confidence level of $95 \%$, and the statistical characteristic and test results of the VaR values are shown in TABLE V.

TABLE V.

THE VAR VALue BASED ON THE GARCH MODEL

\begin{tabular}{|c|c|l|l|l|l|l|l|}
\hline Model & Distribution & Minimum & Maximum & Mean & Std. Dev. & Failure rate & LR statistics \\
\hline \multirow{3}{*}{ GARCH } & normal & 0.011259 & 0.019789 & 0.013585 & 0.001454 & 0.056452 & 0.36574 \\
\cline { 2 - 8 } & $\mathrm{t}$ & 0.012027 & 0.021265 & 0.014492 & 0.001542 & 0.043779 & 0.368458 \\
\cline { 2 - 8 } & GED & 0.011371 & 0.019824 & 0.013632 & 0.001424 & 0.0553 & 0.24845 \\
\hline \multirow{3}{*}{ GARCH-M } & normal & 0.011737 & 0.025111 & 0.014344 & 0.00192 & 0.047235 & 0.071107 \\
\cline { 2 - 8 } & $\mathrm{t}$ & 0.012807 & 0.02783 & 0.015527 & 0.002079 & 0.036866 & 1.727288 \\
\cline { 2 - 8 } EGARCH & GED & 0.011921 & 0.025899 & 0.014479 & 0.001952 & 0.044931 & 0.242704 \\
\cline { 2 - 8 } & normal & 0.010639 & 0.019092 & 0.013572 & 0.001474 & 0.056452 & 0.36574 \\
\cline { 2 - 8 } & GED & 0.011355 & 0.020303 & 0.014457 & 0.001534 & 0.046083 & 0.1438 \\
\hline
\end{tabular}

Can be seen from the TABLE V, the mean and variance of VaR values models calculated by different models have no obvious difference. Under the same distributional hypothesis, the VaR value of GARCH-M model best reflect the risk level of Growth Enterprise Market; under the premise of the same model, the VaR value based on the $t$ distribution assumption has the largest mean and variance.

In terms of the test accuracy of VAR value, the failure rate of each model is not obvious difference and close to $5 \%$, among which the failure rate of GARCH-M model under the $\mathrm{t}$ distribution assumption is the lowest. All the LR statistics calculated from each model are less than the critical value (3.841) of $\chi^{2}$ distribution under the $5 \%$ significance level and one degree of freedom. Therefore, we cannot reject the null hypothesis under the $5 \%$ significance level, which means that the VaR value based on the GARCH model group accurately reflects the risk level of the Growth Enterprise Market.

\section{CONCLUSION}

This paper uses the latest data of GEM to measure the volatility of China's GEM market risk at a certain confidence level by the VaR and GARCH model. Through the analysis of sample data, conclusions could be drawn that the GEM returns sequence has the characteristic of sharp peaks and fat tail; In GARCH model, the GARCH coefficients are between 0.9 and 1 , which means the GEM composite index has long-term memory, and the past price volatility has close relationship with the future price. In the model results, ARCH and GARCH coefficients are positive, which means the past volatility has the positive effect on the future, which causes volatility cluster.

Under the hypothesis of same distribution, the VaR values based on GARCH-M and EGARCH model are better than GARCH model, for they consider the features of the earnings is proportional to the risk and leverage effect respectively. In this article, it's clear that the 
GARCH-M model is the best one in the calculation of VaR.

In the case of using the same model, the VAR values calculated based on the fat tail distributions of $t$ and GED distribution are better the normal distribution, because they consider the characteristics of volatility cluster and fat tail. Obviously, the $t$ distribution is the most suitable one for the risk measurement of GEM based on the method of VaR in China.

\section{REFERENCES}

[1] Wang Chunfeng, Wan Haihui, Zhang Wei, "The model of market risk measurement——VaR," Journal of Systems Engineering, Vol. 15 , pp.67-85, 2000.

[2] Dai Guoqiang, Xu Longbing, Lu Rong, "The reference and application of VaR method to the financial risk management in our country," Journal of Financial Research, Vol. 7, pp.45-51, 2000

[3] Xu Wei, Huang Yanlong, "Empirical analysis on GARCH-type models and VaR," The Research of Quantitative and Technical Economics, vol. 1, 2008, pp. 120-132.

[4] Gong Rui, Chen Zhongchang, Yang Dongrui, "To Evaluate VaR of China Stock Marketing Comparatively by Using GARCH Family Model and Comment," The Research of Quantitative and Technical Economics, Vol. 7, 2005, pp.67-81.
[5] Chen Linfen, Wang Dequan, "The research of securities market risk measurement based on the GARCH and VaR methods," Industrial Technology Economy, J. Vol. 28, No.11, 2009, pp.128137.

[6] Zou Jianjun, Zhang Zongyi, Qin Zheng, "The Application of GARCH Model in Computing the VaR of Chinese Stock Market," Theory and Practice of Systems Engineering, vol. 5, 2003, pp. 2025.

[7] Xiao Chunlai, Song Ran, "The theory and application of VaR," Mathematical Statistics and Management, Vol. 22, No2, 2003, pp.6-10.

[8] Chen Shoudong, Yu Shidian, "Analysis of China's stock marke using VaR method based on GARCH model," Jilin University Journal Social Sciences Edition, No.4, 2002, pp.11-17.

[9] Li Yajing, Zhu Hongquan, Peng Yuwei, "Forcasting Chinese stock markets volatility based on GARCH models," Mathematics in Practice and Theory, Vol. 33, No.11, 2003, pp.65-71.

[10] Hu Jingsheng, Wang Rong, Ding Wei, "Application of the VaR method and its extended models in portfolio risk management," The Research of Quantitative and Technical Economics, No. 5, 2005, pp.141-150.

[11] Chen Shufang, Chen Shengshuang, "VaR measurement of market risk based on GARCH family model," Journal of WUT (Information and Management Engineering), Vol. 34, No. 6, 2012, pp.767-771. 